\section{Use of an ATP \\ bioluminescence meter to quantify and monitor practice cleanliness}

\section{Katie Whalley, Martin Whitehead}

Chipping Norton Veterinary Hospital, Chipping Norton, Oxfordshire, United Kingdom

\section{OBJECTIVES}

Veterinary practices typically assess cleanliness in clinical areas by visual inspection - an unreliable method. Objective methods for quantifying cleanliness have been developed for human hospitals. One is measurement of ATP on surfaces - the amount of ATP correlates to the amount of organic matter present and so to cleanliness. We assessed the use of a commercially available ATP bioluminescence meter (Hygiena SystemSURE Plus) - that provides an immediate (15 seconds) objective measurement of cleanliness - in the clinical areas of our hospital before and after a change in our routine cleaning protocol.

\section{METHODS}

22 fixed surfaces or items of equipment in the dog and cat wards, prep room, ultrasound room and theatres were swabbed shortly after routine cleaning towards the end of the day, twice a week on 11 occasions, both before and after the change in cleaning protocol. The nursing staff doing the cleaning did not know which surfaces were being tested and were not made aware of the results until the end of the study.

\section{RESULTS}

Before changing our cleaning protocol, ATP measurements in most locations were above - for some sites, far above - thresholds recommended in human hospitals, despite our clinical areas appearing clean by visual inspection. After changing our cleaning protocol, measurements overall greatly decreased, indicating improved cleanliness. Median measurements decreased $>20 \%$ at 19 of the 22 sites tested, and increased $>20 \%$ at only one site.

\section{STATEMENT (CONCLUSIONS)}

These findings demonstrate that ATP bioluminescence is a superior method to visual inspection for assessing cleanliness - as previously demonstrated in human hospitals.

\section{Benchmarking antibiotic prescribing by individual veterinary surgeons within a practice}

\section{Martin Whitehead}

Chipping Norton Veterinary Hospital, Chipping Norton, United Kingdom

\section{OBJECTIVES}

Many NHS health centres regularly count antibiotic prescriptions by each GP doctor, allowing comparison between GPs in a practice, and across practices, for benchmarking purposes - helping reduce antimicrobial usage, and thereby antimicrobial resistance. This method was applied to the nine permanent veterinary surgeons in one small-animal veterinary hospital.

\section{METHODS}

Prescriptions of preparations containing antibiotics (doses and course durations were ignored) booked on the hospital's practice management system between
1/1/2018 - 30/6/2019 were retrospectively counted for each permanent vet (locums and visiting specialists excluded), for each of dogs and cats (other species excluded). Each vets' number of prescriptions was expressed as a percentage of the number of dog or cat consults (excluding vaccination consults) by that vet, to normalise for differences in the amount of dog or cat work across vets.

\section{RESULTS}

Despite broadly similar general-practice case portfolios, the total number of antibiotic prescriptions, and the relative proportions of different antibiotic types - including 'critically-important' antibiotics - prescribed, varied substantially between individual vets. For each of dogs and cats, for each of systemic courses (tablets, oral drops, or injectable cefovecin), skin creams, eardrops, and topical eye preparations containing antibiotics, the number of prescriptions varied across vets by a factor of $>2$ to $>4$, i.e., depending on treatment category, some vets prescribed antibiotics $>100 \%$ to $>300 \%$ more often than others. Higher prescribers for dogs tended to be higher prescribers for cats also.

\section{STATEMENT (CONCLUSIONS)}

These results indicate substantial scope to reduce antibiotic usage by adjusting the prescribing behaviour of higher-prescribing vets to match that of lowerprescribing vets. 